\title{
Identification of key genes associated with the progression of intrahepatic cholangiocarcinoma using weighted gene co-expression network analysis
}

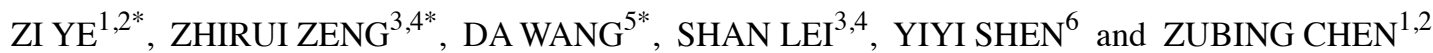 \\ Departments of ${ }^{1}$ General Surgery and ${ }^{2}$ Hepatobiliary Surgery, Renmin Hospital of Wuhan University, Wuhan, \\ Hubei 430060; ${ }^{3}$ Guizhou Provincial Key Laboratory of Pathogenesis and Drug Research on Common Chronic Diseases; \\ ${ }^{4}$ Department of Physiology, School of Basic Medicine, Guizhou Medical University, Guiyang, Guizhou 550009; \\ ${ }^{5}$ Department of Biliary-Pancreatic Surgery, Tongji Hospital, Tongji Medical College, Huazhong University of Science and \\ Technology, Wuhan, Hubei 430060; ${ }^{6}$ Department of Liver-Biliary Surgery, Affiliated Hospital of Guizhou \\ Medical University, Guiyang, Guizhou 550009, P.R. China
}

Received April 6, 2019; Accepted October 22, 2019

DOI: $10.3892 / \mathrm{ol} .2020 .11600$

\begin{abstract}
The present study aimed to identify the key genes that are associated with the progression of intrahepatic cholangiocarcinoma through weighted gene co-expression network analysis (WGCNA). A total of three gene datasets were downloaded from the Gene Expression Omnibus database, including GSE107943, GSE119336 and GSE26566. Differentially expressed genes (DEGs) between intrahepatic cholangiocarcinoma tissues and adjacent liver tissues were identified using GSE107943, while tissue specific genes between bile duct and liver tissues were identified using GSE26566. Following the removal of tissue-specific genes, real DEGs were used to construct the WGCNA to investigate the association between gene modules and clinical traits. Following functional analysis, pathway enrichment analysis and the construction of a protein-protein interaction (PPI) network were performed, hub genes were selected and their diagnostic value was verified in GSE119336 using a receiver operating characteristic curve. Finally, the protein levels of the hub genes were also verified in intrahepatic cholangiocarcinoma tissues. A total of 1,643 real DEGs were identified and used to construct the WGCNA. Additionally, a total of seven co-expressed gene modules were identified following WGCNA, while genes in brown and yellow modules were identified to be associated with multiple clinical traits (the
\end{abstract}

Correspondence to: Professor Zubing Chen, Department of Hepatobiliary Surgery, Renmin Hospital of Wuhan University, 99 Zhangzhi Dong Road, Wuhan, Hubei 430060, P.R. China

E-mail: chenzubing@aliyun.com

${ }^{*}$ Contributed equally

Key words: intrahepatic cholangiocarcinoma, weighted gene co-expression network analysis, hub genes number of clinical traits $>3$ ) and used as key modules. A total of 63 core key module genes were subsequently identified, and it was indicated that these genes were most enriched in the nucleus (Gene Ontology term) and the cell cycle pathway (Kyoto Encyclopedia of Genes and Genomes term). Finally, a total of eight genes, including cyclin B1, cell division cycle 20 , cell division cycle associated 8 , cyclin dependent kinase 1 , centrosomal protein 55 , kinesin family member $2 \mathrm{C}$, DNA topoisomerase II $\alpha$ and TPX2 microtubule nucleation factor, exhibited the highest score in PPI analysis and had a high diagnostic value for intrahepatic cholangiocarcinoma. In addition, the protein levels of these genes were also revealed to be increased in most intrahepatic cholangiocarcinoma tissues. These eight genes may be used as novel biomarkers for the diagnosis of intrahepatic cholangiocarcinoma.

\section{Introduction}

Among the numerous types of primary liver malignancy, intrahepatic cholangiocarcinoma accounts for $\sim 15 \%$ of cases globally. In previous years, the incidence of intrahepatic cholangiocarcinoma has risen, and this rise is anticipated to increase further (1). However, $>50 \%$ of patients who undergo excision exhibit disease recurrence, which most commonly occurs in the remnant liver (2). The molecular mechanisms of the progression of intrahepatic cholangiocarcinoma have yet to be determined. Therefore, it is necessary to identify the molecular mechanisms associated with the development of intrahepatic cholangiocarcinoma.

High-throughput microarray and RNA sequencing technology, which are able to identify changes in gene expression, have been widely used in studying the etiology of numerous cancer types, including hepatocellular carcinoma (3), breast cancer (4) and pancreatic cancer (5). Screening novel biomarkers for tumor diagnosis and the pathways associated with tumor development, recurrence and drug resistance, are also critical. Using bioinformatics analysis, Xiao et al (6) revealed five potential biomarkers that serve a key function in 
the progression of adrenocortical carcinoma and are associated with a poor outcome. Similarly, through integrated analysis, Huang et al (7) investigated five genes that were indicated to contribute to multidrug resistance in patients with Hodgkin's lymphoma. However, the molecular mechanisms associated with the progression of intrahepatic cholangiocarcinoma are yet to be determined.

In the present study, GSE107943 was used as a discovery cohort to identify differentially expressed genes (DEGs) and perform weighted gene co-expression network analysis (WGCNA), while GSE26566 was used to identify tissue-specific genes. Furthermore, GSE119336 was used as a validation cohort. Subsequent to the removal of tissue-specific genes, real DEGs were used to construct the WGCNA. Followed by Gene Ontology (GO) enrichment analysis, Kyoto Encyclopaedia of Genes and Genomes (KEGG) analysis, protein-protein network (PPI) interactions, receiver operating characteristic curve (ROC) analysis and immumohistochemical staining on intrahepatic cholangiocarcinoma tissues, the hub genes were identified. These hub genes may be used for future developments for the diagnosis and treatment of intrahepatic cholangiocarcinoma.

\section{Materials and methods}

Data processing. Gene expression profile data GSE107943, GSE119336 and GSE26566 were obtained from the Gene Expression Omnibus (http://www.ncbi.nlm.nih.gov/geo) (8). A total of 27 adjacent tissues and 30 intrahepatic cholangiocarcinoma tissues were included in the GSE107943 profile. A total of 15 adjacent tissues and 15 intrahepatic cholangiocarcinoma tissues were included in the GSE119336 profile. A total of 59 normal liver tissues and 6 normal bile duct tissues were included in the GSE26556 profile. The GSE107943 array data were acquired from Illumina NextSeq 500 (Homo sapiens; GPL18573). The GSE119336 array data were acquired from Illumina HiSeq 2000 (Homo sapiens; GPL11154). The GSE26566 array data were acquired from Illumina humanRef-8 v2.0 expression beadchip (GPL6104). The gene expression profile data were normalized using $\mathrm{R}$ software (version: 3.5.2) prior to DEG analysis.

DEG analysis. The R software was used to identify DEGs in 27 adjacent non-tumor tissues and 30 intrahepatic cholangiocarcinoma tissues. The tissue-specific genes between 59 normal liver tissues and 6 normal bile duct tissues were also analyzed. A $\mid \log 2$ fold change (FC) $\mid \geq 2$ and an adjusted value of $\mathrm{P}<0.05$ were considered to indicate a statistically significant difference. The expressions of all genes are presented in a volcano plot, while the expression of DEGs in each sample is presented in a heatmap. Following the removal of tissues-specific genes, real DEGs were obtained and included in the WGCNA analysis.

Construction of WGCNA. The R package 'WGCNA' was used in the present study to construct a co-expression network for the real DEGs identified in the 30 intrahepatic cholangiocarcinoma samples. To construct the WGCNA, the expression profile of DEGs and their clinical trait information were imported into R software. A Pearson's correlation analysis was subsequently performed to cluster samples and detect outliers. The threshold for identifying outlier samples was 80 cut-height, and the results identified no outlier samples (Fig. S1). All gene pairs were then analyzed using Pearson's correlation analysis, and a matrix of similarity was constructed based on this analysis. Subsequently, to achieve a scale-free co-expression network, the matrix of similarity was constructed using a soft power of $\beta=5$. The adjacency matrix was then translated into a topological overlap matrix (TOM). Furthermore, the median linkage hierarchical clustering was analyzed using the TOM-based dissimilarity measure with the mini-size set as 30 .

Identification of clinically significant modules and verification of module hub genes. Following the construction of the WGCNA, different module eigengenes and their corresponding clinical traits were correlated. These traits included the recurrence, disease free survival in months, tumor associated mortality, overall survival in months, carcinoembryonic antigen expression, carbohydrate antigen 19-9 expression, tumor differentiation, tumor class (Child-Pugh score) (9), American Joint Committee on Cancer stage (10), tumor size, vascular invasion and hepatitis B infection. Modules were considered relevant to the clinical phenotype if their correlation score with clinical traits was $>0.3$ and if they exhibited a $\mathrm{P}<0.05$ statistically significant difference. Modules associated with multiple clinical traits (the number of clinical traits $>3$ ) were set as key modules. These modules were then selected and enrolled in further analysis. The gene significance (GS), which was quantified using associations between individual genes and the clinical trait of interest, and the module membership (MM), which was the correlation between the module eigengenes and the gene expression profiles, were calculated. MM was defined as $>0.8$ and GS was defined as $>0.2$ for screening module core genes. If modules were associated with numerous clinical traits, the Bioinformatics \& Evolutionary Genomics Venn Diagram tool (http://bioinformatics.psb.ugent.be/webtools/Venn/) was used to locate intersected module core genes.

GO and pathway enrichment analysis. DAVID 6.8 (http://david-d.ncifcrf.gov/) $(11,12)$ was used in the present study to perform functional enriched analysis and pathway enrichment analysis for the module core genes, including in GO (biological process, molecular function and cellular component) and KEGG pathway analysis. $\mathrm{P}<0.05$ was considered to indicate a statistically significant result. The results of GO and KEGG analysis were visualized using R software.

Construction of the PPI network. STRING (available online: http://string-db.org) (13) was used in the present study to construct the PPI network, and the node and edge information was exported into a text file. Cytoscape software (version: 6.1; https://cytoscape.org/) was used to visualize the PPI network and analyze the combined degree of module hub genes (14). Finally, degree analysis was performed and genes with the highest degree score were identified as hub genes in the PPI network.

Diagnostic value of hub genes. The sensitivity and specificity of the hub genes identified in the present study were evaluated 
through the ROC curve analysis based on the gene expression profile GSE119336. The ROC curve was created using SPSS 20.0 (IBM Corp., Armonk, NY, USA). Genes exhibiting an area under the curve (AUC) value of $>0.7$ were considered to exhibit a good diagnostic value.

Tissues ethics. The procedures of the present study were ethically approved by the Clinical Ethics Management Committee of Renmin Hospital of Wuhan University. A total of 23 intrahepatic cholangiocarcinoma tissues and corresponding adjacent tissues were obtained between April 2019 and August 2019. None of the patients enrolled in the present study received neoadjuvant chemotherapy, radiotherapy or immunotherapy prior to surgery. Similarly, these patients had no other systemic diseases, such as diabetes mellitus and rheumatic disease. All patients (mean age, 54.3 \pm 9.2 , range, 41-69 years; 10 males and 13 females) who provided tissue samples provided written informed consent.

Immumohistochemical staining. All tissues were fixed in $4 \%$ paraformaldehyde for $30 \mathrm{~min}$ under room temperature and sectioned into 4- $\mu \mathrm{m}$ thick paraffin-embedded sections. After heating at $60^{\circ} \mathrm{C}$ for $1 \mathrm{~h}$, the specimens were deparaffinized using xylene under room temperature and rehydrated with graded ethanol $(100,80,60$ and 40\%). Subsequent to antigen retrieval with sodium citrate $(100 \mathrm{mM})$, the sections were blocked using $3 \% \mathrm{H}_{2} \mathrm{O}_{2}$ for $20 \mathrm{~min}$ and $5 \%$ bovine serum albumin (Wuhan Servicebio Technology Co., Ltd., Wuhan, China) for 30 min under room temperature. The specimens were then incubated with the primary antibodies, including CCNB1 (1:400; cat. no. A16800; ABconal Biotech Co., Ltd.), CDC20 (1:400; cat. no. A15656; ABconal Biotech Co., Ltd.), CDCA8 (1:400; cat. no. A12594; ABconal Biotech Co., Ltd.), CDK1 (1:400; cat. no. A2861; ABconal Biotech Co., Ltd.), CEP55 (1:500; cat. no. 23891-1-AP; ProteinTech Group, Inc.), KIF2C (1:400; cat. no. A5449; ABconal Biotech Co., Ltd.), TOP2A (1:200; cat. no. A0726; ABconal Biotech Co., Ltd.) and TPX2 (1:200; cat. no. A4522; ABconal Biotech Co., Ltd.) for $12 \mathrm{~h}$ at $4^{\circ} \mathrm{C}$. Subsequently, the sections were immunohistochemically stained with horseradish peroxidase (HRP)-conjugated secondary goat anti-mouse and -rabbit antibodies (cat. no. G1210-2-A-100; dilution: 1:200; Wuhan Servicebio Technology Co., Ltd.) for $2 \mathrm{~h}$ at room temperature. Subsequent to incubation with the Cell and Tissue Staining HRP-3,3'-diaminobenzidine kit (Wuhan Servicebio Technology Co., Ltd.), an orthophotomicroscope was used to collect images. Finally, the protein levels of the target genes were evaluated according to the sum of the intensity and percentage scores. Stain intensity score: 0 (no staining), 1 $(+), 2(++)$ and $3(+++)$; the proportion of positive cells score: $0(0-1 \%), 1(1-33 \%), 2(34-66 \%)$ and $3(67-100 \%)$. The total staining scores were defined as follows: 0-2 (low expression); 3-4 (moderate expression); 5-6 (high expression). The intensity and percentage scores were determined using Image pro plus software (version number: 6.0; Media Cybernetics, Inc.).

\section{Results}

Identification of real DEGs. A GSE107943 profile including 30 intrahepatic cholangiocarcinoma tissues and 27 adjacent non-tumor tissues was used to identify DEGs. DEGs were identified using R software and the cutoff was set as $\mid \log 2 \mathrm{FCl} \geq 2$ and an adjusted P-value $<0.05$. A total of 1,675 DEGs were identified between intrahepatic cholangiocarcinoma and the adjacent non-tumor tissues, including 1,129 upregulated DEGs and 546 downregulated DEGs (Fig. 1A and B). The adjacent tissues originated from liver tissues, therefore, genes known to be differentially expressed between liver and bile duct tissues needed to be removed. The gene expression profile of GSE26566 was used to identify specific genes that differed between liver and bile duct tissues. The results indicated that 153 genes were upregulated in normal bile duct tissues and 3 genes were upregulated in normal liver tissues (Fig. 1C). Subsequent to removing tissues-specific genes which were contained in the DEGs indicated by the analysis of GSE107943, the 1,643 real DEGs, which included 1,098 real upregulated DEGs and 545 real downregulated DEGs, were identified (Fig. 1D). All real DEGs were subsequently included in the WGCNA analysis.

WCGNA construction. To construct the WGCNA, a soft power of $\beta=5$ (scale-free $R^{2}=0.88$ ) was selected as the soft threshold to ensure a scale-free network (Fig. 2A-D). A total of seven co-expressed modules were identified (black, brown, blue, yellow, green, red and turquoise), while genes with no cluster were distributed in the grey module (Fig. 2E). The seven co-expressed networks were then used in further analysis.

Identification of significant modules and module core genes. The results revealed five modules that were significantly associated with clinical traits in patients with intrahepatic cholangiocarcinoma, including black, brown, blue, yellow and red modules. Genes in blue and black modules were significantly associated with tumor class $(\mathrm{R}=0.41, \mathrm{P}=0.02 ; \mathrm{R}=0.71$, $\mathrm{P}=1 \times 10^{-05}$; respectively), while genes in red modules were significantly associated with tumor class $\left(\mathrm{R}=-0.74, \mathrm{P}=3 \times 10^{-06}\right)$ and $\mathrm{HBV}$ infection $(\mathrm{R}=0.41, \mathrm{P}=0.02)$. Genes in brown modules were significantly associated with tumor recurrence $(\mathrm{R}=0.52$, $\mathrm{P}=0.003)$, tumor-associated mortality $(\mathrm{R}=0.47, \mathrm{P}=0.009)$, serum carbohydrate antigen 19-9 (CA19-9) expression $(\mathrm{R}=0.45$, $\mathrm{P}=0.01)$ and tumor class $(\mathrm{R}=0.49, \mathrm{P}=0.006)$. Furthermore, genes in yellow modules were significantly associated with recurrence $(\mathrm{R}=0.49, \mathrm{P}=0.006)$, disease-free survival in months $(\mathrm{R}=-0.41, \mathrm{P}=0.02)$, tumor-associated mortality $(\mathrm{R}=0.52$, $\mathrm{P}=0.003)$ and tumor class $(\mathrm{R}=0.83, \mathrm{P}=2 \mathrm{e}-08$; Fig. 3$)$. Due to genes in brown and yellow modules being significantly associated with the number of clinical traits $(>3)$, these modules were regarded as key modules and used in the subsequent analysis. The MM in the brown module and the gene significance for tumor recurrence, mortality, serum CA199 expression and tumor class exhibited a high correlation (cor $=0.5$ for recurrence; cor $=0.31$ for tumor-associated mortality; cor $=0.45$ for CA199 expression; cor $=0.38$ for tumor class; Fig. 4A-D). Furthermore, a total of 38 genes were demonstrated to meet the threshold in the brown module for the four clinical traits, and these 38 genes were identified as core genes (Fig. 4E). Additionally, the $\mathrm{MM}$ in the yellow module and the gene significance for tumor recurrence, disease-free survival in months, tumor-associated mortality and tumor class also exhibited a high correlation (cor=0.59 for recurrence; cor=0.29 for disease-free survival 
A

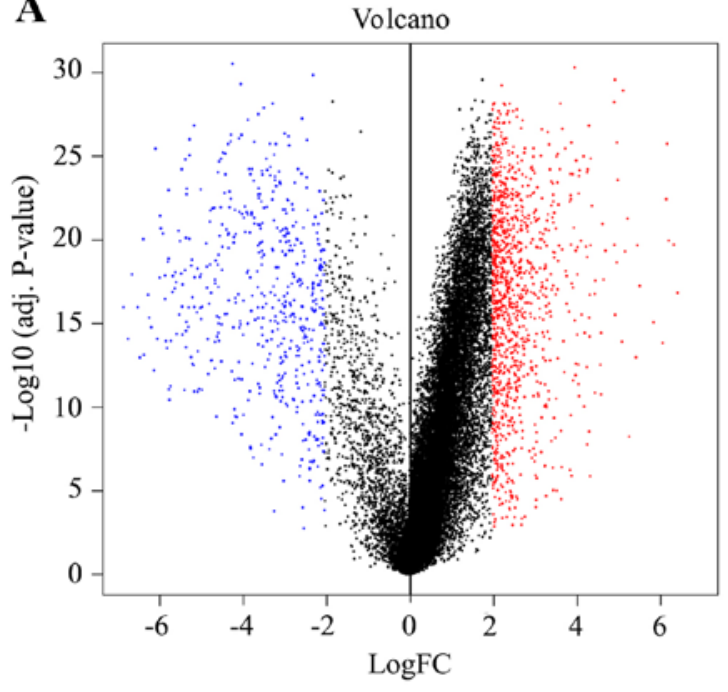

B

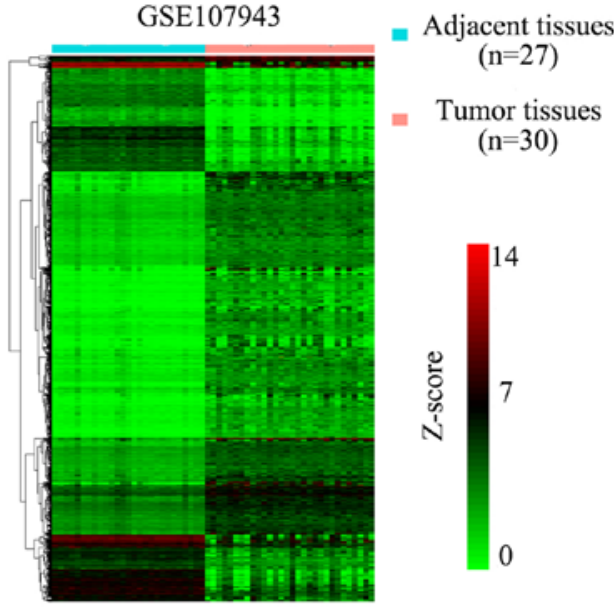

C

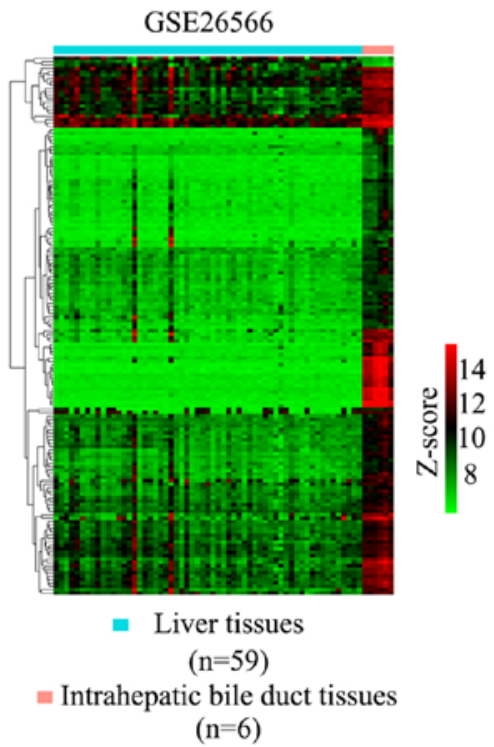

D

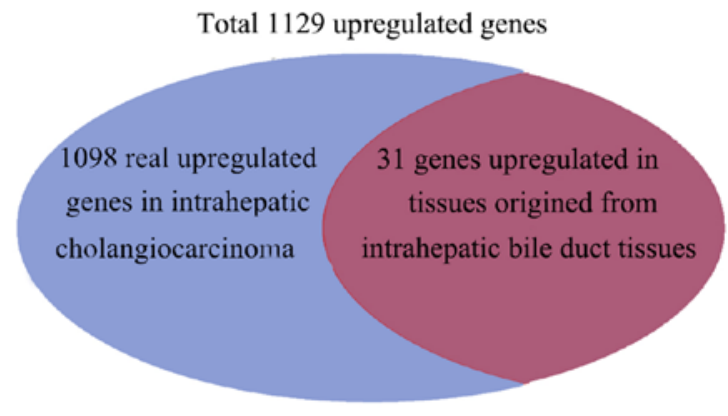

$\mathbf{E}$

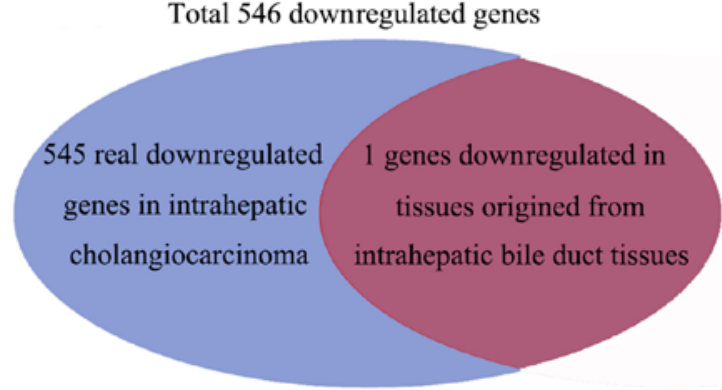

Figure 1. Identification of real DEGs in intrahepatic cholangiocarcinoma. (A) A volcano plot revealing the expression of all genes in GSE107943. The red dots represent all upregulated DEGs, and blue dots represent all downregulated DEGs. (B) A heatmap chart revealing the expression of each DEG in each sample in GSE107943. Green indicates downregulated, while red indicates upregulated. (C) A heatmap chart revealing DEGs (also named tissue-specific genes) between normal liver tissues $(n=59)$ and normal bile duct tissues $(n=6)$. Venn diagram indicating the real DEGs that were (D) upregulated or $(E)$ downregulated in intrahepatic cholangiocarcinoma. DEG, differentially expressed genes; FC, fold change.

in months; cor $=0.55$ for tumor-associated mortality; cor $=0.85$ for tumor class; Fig. 4F-I). Furthermore, a total of 25 genes in the yellow module met the threshold for the four clinical traits, and these 25 genes were identified as core genes (Fig. 4J). Therefore, a total of 63 core genes in the brown and yellow modules were used in further analysis.

Functional and pathway analysis of core genes. To understand the enriched function and the KEGG pathway in the 63 core genes, the DAVID online tool was used. The results demonstrated that the top five biological processes that were associated with these 63 core genes were mitotic spindle assembly, mitotic metaphase plate congression, positive regulation of mitosis, mitotic sister chromatid segregation and the spindle checkpoint (Fig. 5A). Only three molecular function terms were indicated, and these were genes that were enriched in ATP binding, chromatin binding and DNA binding (Fig. 5B). Furthermore, the top five cellular components which were associated with these genes were the nucleus, cytoplasm, nucleoplasm, midbody and cell membrane (Fig. 5C). Furthermore, the results revealed that the core genes were enriched in five pathways: The cell cycle pathway, oocyte meiosis pathway, p53 signaling pathway, insulin secretion pathway and progesterone-mediated oocyte maturation pathway (Fig. 5D).

Construction of PPI network and verification of hub genes. To identify hub genes in intrahepatic cholangiocarcinoma, a total of 63 module core genes were used to construct the PPI network. A total of 56 nodes and 512 edges were indicated in the network (Fig. 6A). Furthermore, the results revealed that CCNB1, CDC20, CDCA8, CDK1, CEP55, KIF2C, TOP2A 

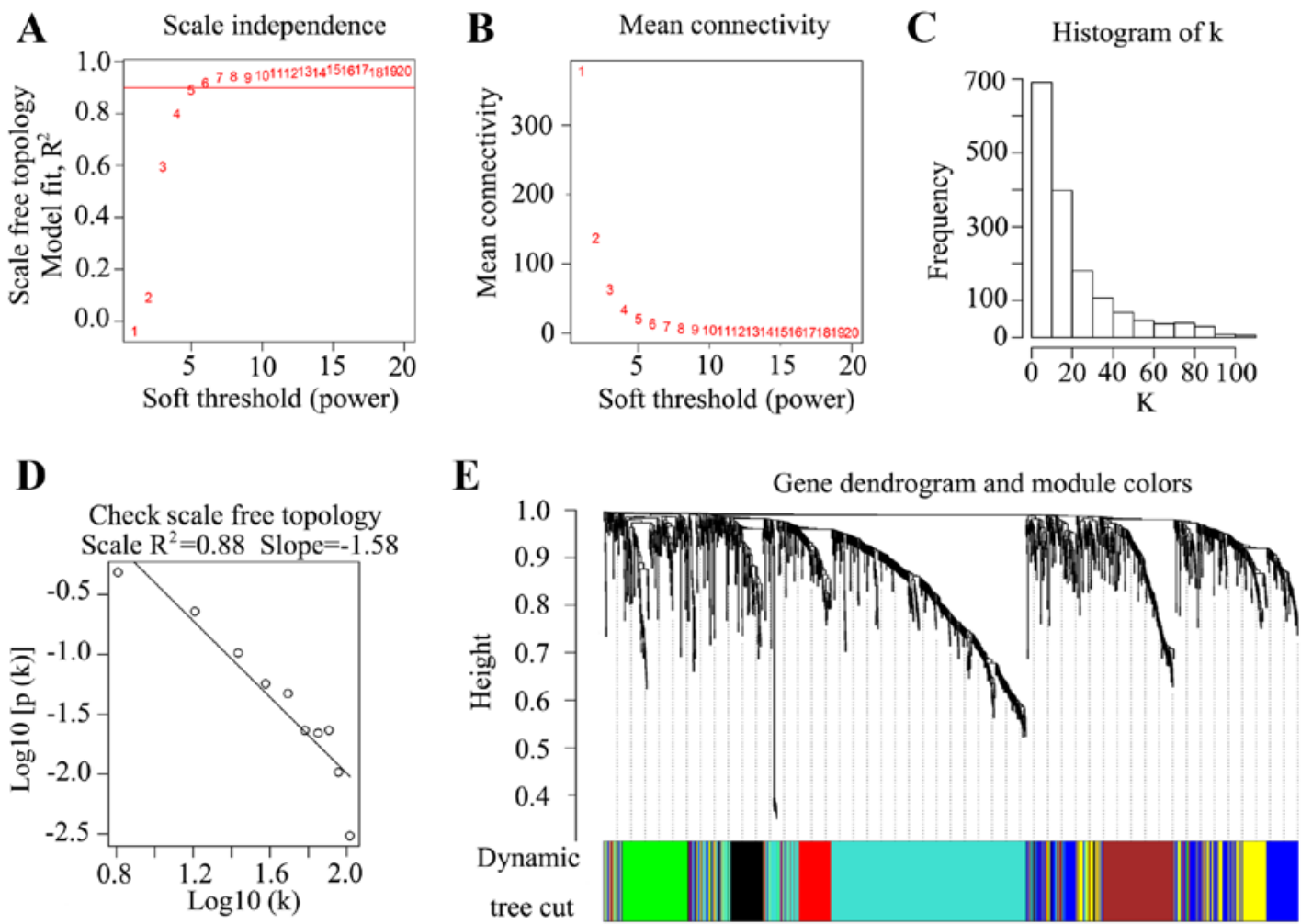

Figure 2. Selection of threshold and construction of weighted gene co-expression network analysis. (A) Scale independence. (B) Mean connectivity of various soft-thresholding values ( $\beta$ ). (C) Histogram of connectivity distribution when $\beta=5$. (D) Checking the scale-free topology when $\beta=5$. (E) Dendrogram of all differentially expressed genes clustered based on a dissimilarity measure (1-topological overlap matrix). A total of seven co-expressed modules were identified (black, brown, blue, yellow, green, red and turquoise), while genes exhibiting no clusters were distributed in the grey modules.

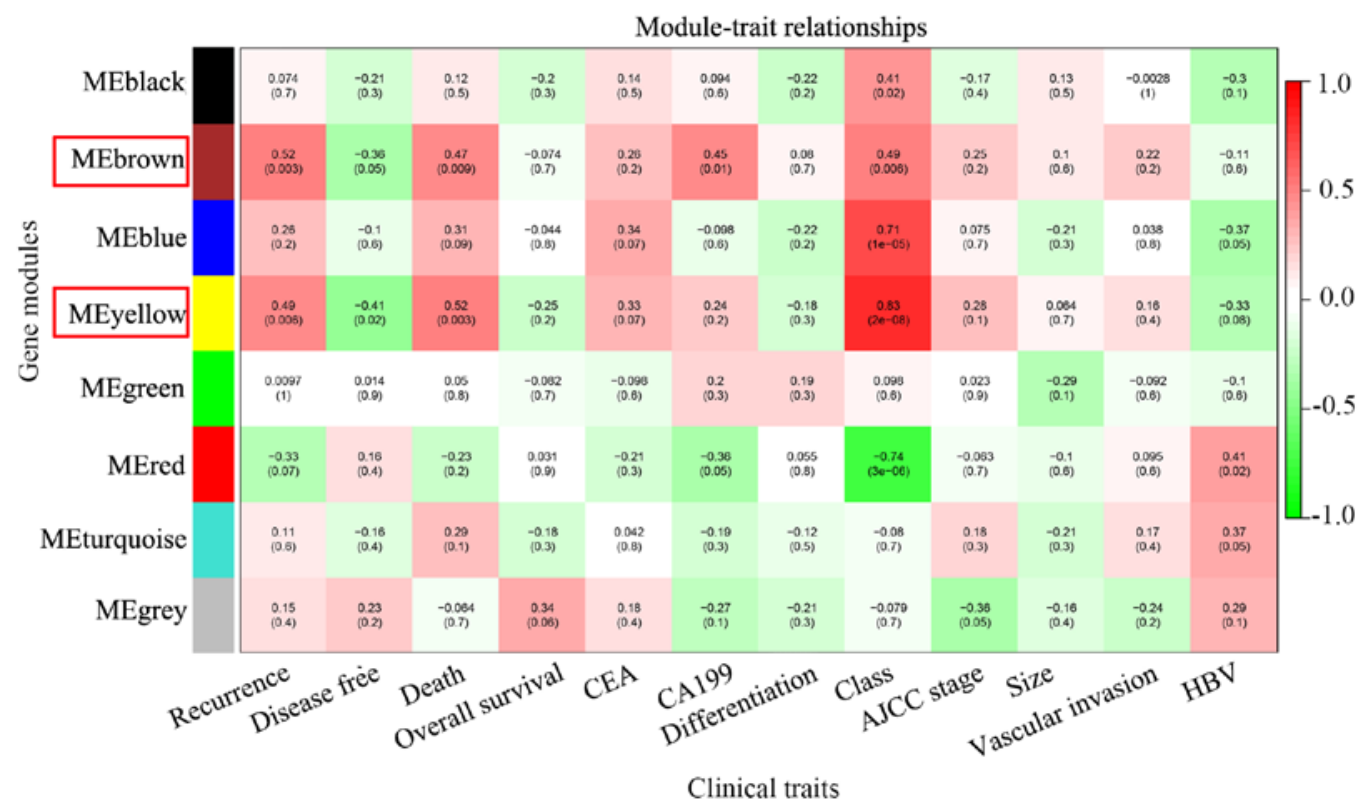

Figure 3. Identification of key modules. Heatmap of the correlation between module eigengenes and clinical traits of intrahepatic cholangiocarcinoma. A total of 12 clinical traits were included: Recurrence, disease-free survival months, tumor-associated mortality, overall survival months, CEA expression, CA199 expression, tumor differentiation, tumor class (Child-Pugh score), AJCC stage, tumor size, vascular invasion and HBV infection. Brown and yellow modules were associated with multiple clinical traits (the number of clinical traits $>3$ ), and they were regarded as key modules and highlighted with a red frame. HBV, hepatitis B virus; AJCC, American Joint Committee on Cancer; CEA, carcinoembryonic antigen; CA199, carbohydrate antigen 19-9.

and TPX2 exhibited the highest degree score in the PPI network and these genes were subsequently identified as hub genes (Fig. 6B).
Diagnosis value of hub genes. In order to detect the diagnostic value of these eight hub genes, the gene expressed profile GSE119336, including 15 adjacent tissues and 15 intrahepatic 
A

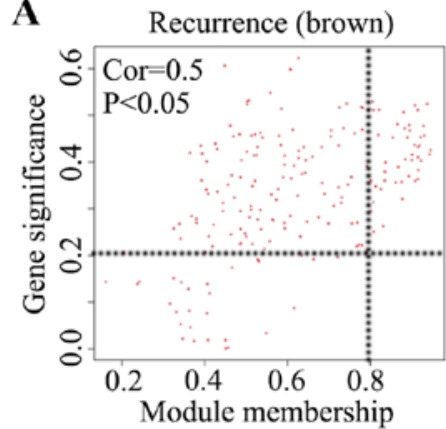

B

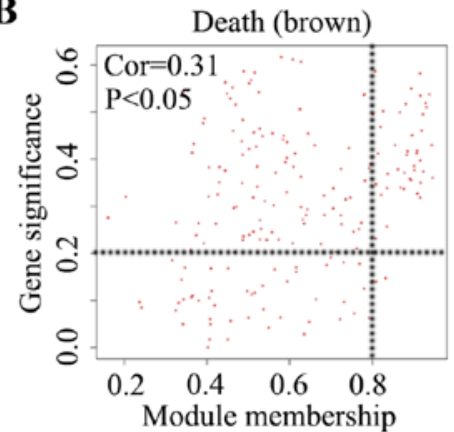

C

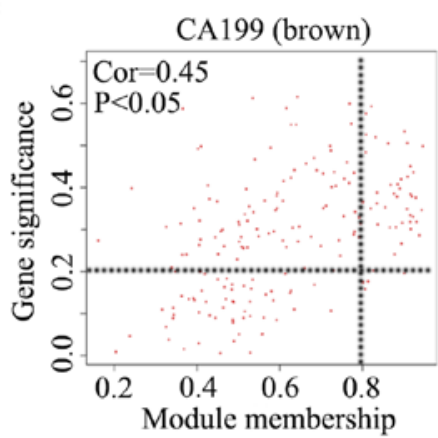

D

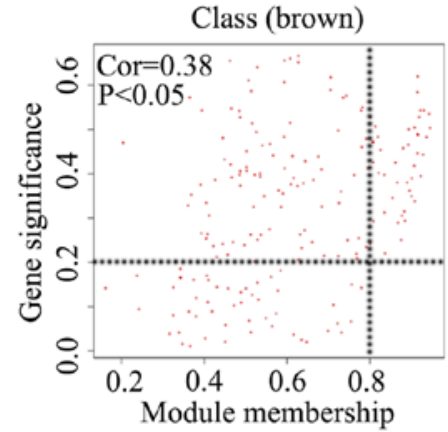

$\mathbf{E}$

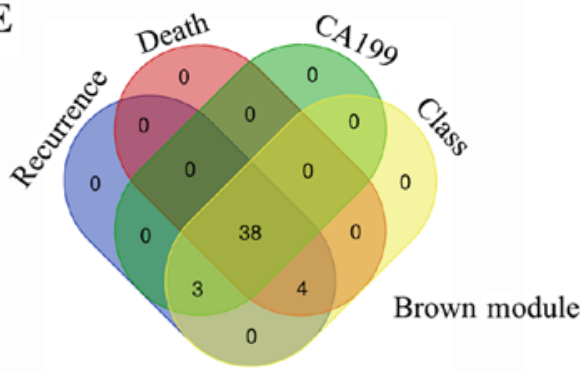

F

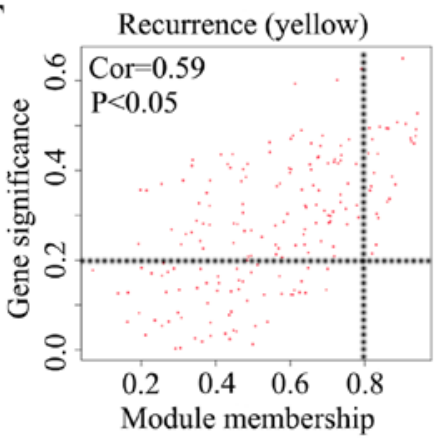

G

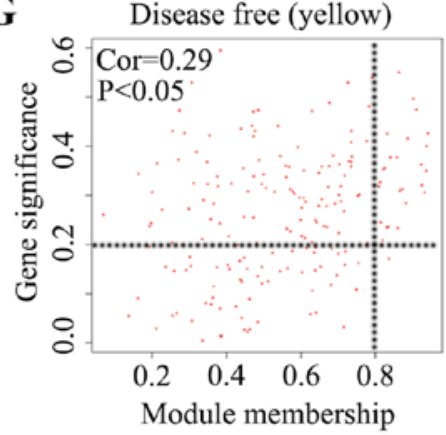

$\mathbf{H}$

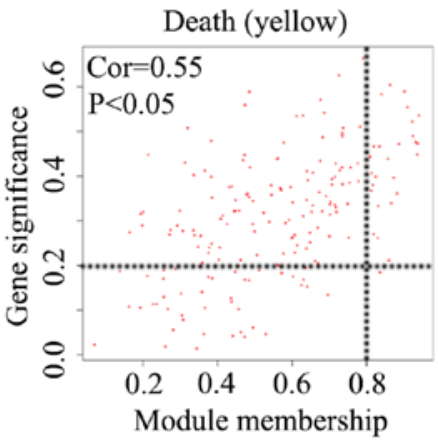

I

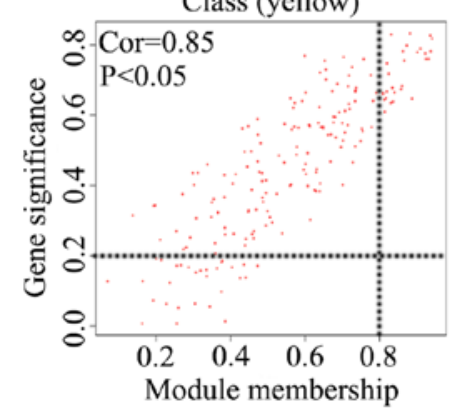

$\mathbf{J}$

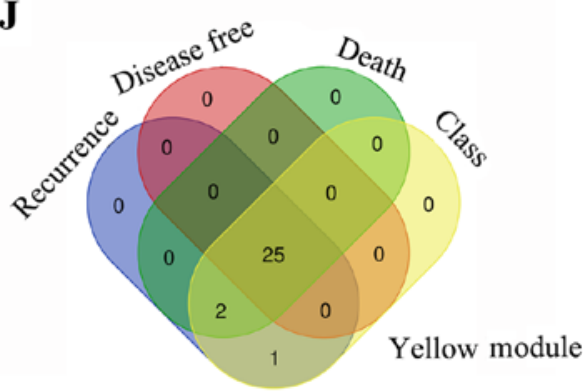

Figure 4. Genes associated with the clinical traits of intrahepatic cholangiocarcinoma and identification of core genes. Scatter plot of module eigengenes associated with (A) tumor recurrence, (B) tumor-associated mortality, (C) serum CA199 expression and (D) tumor class (Child-Pugh score) in the brown module. Genes with a module membership $>0.8$ and a gene significance for clinical trait of $>0.2$ were regarded as module core genes. (E) Venn diagram to identify intersectional module core genes which are strongly associated with tumor recurrence, tumor-associated mortality, serum CA199 expression and tumor class (Child-Pugh score) in the brown module. (F-I) Scatter plot of module eigengenes associated with (F) tumor recurrence, (G) disease-free survival months, (H) tumor-associated mortality and (I) tumor class (Child-Pugh score) in the yellow module. Genes with a module membership $>0.8$ and a gene significance for clinical trait $>0.2$ were regarded as module core genes. (J) Venn diagram to identify intersectional module core genes which are strongly associated with tumor recurrence, disease-free survival in months, tumor-associated mortality and tumor class (Child-Pugh score) in the yellow module. CA199, carbohydrate antigen 19-9.

cholangiocarcinoma tissues, was used and a ROC analysis was performed (Fig. 7). The results revealed that CCNB1, CDC20, CDCA8, CDK1, CEP55, KIF2C, TOP2A and TPX2 all exhibited good diagnostic values for intrahepatic cholangiocarcinoma, using a cut-off AUC value of 0.7. The results of the AUC were as follows: CCNB1 (AUC $=0.969$ ), CDC20
(AUC=0.964), CDCA8 (AUC=0.960), CDK1 $(\mathrm{AUC}=0.929)$, CEP55 (AUC $=0.964), \mathrm{KIF} 2 \mathrm{C} \quad(\mathrm{AUC}=0.973), \mathrm{TOP} 2 \mathrm{~A}$ $(\mathrm{AUC}=0.942)$ and TPX2 (AUC=0.911).

Verification of the expression of hub genes. In order to verify the results obtained from prediction, immumohistochemical 
A

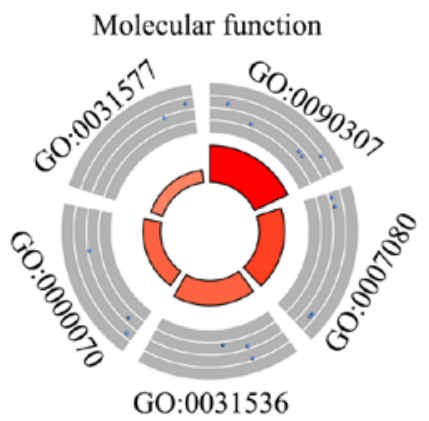

B

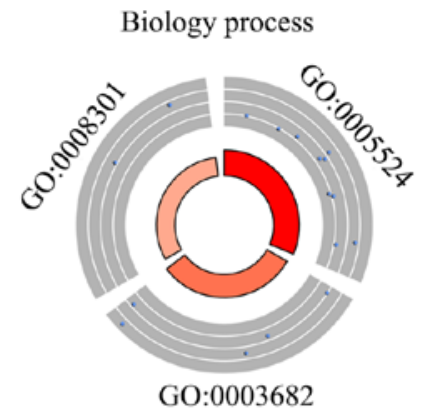

GO:0003682

\begin{tabular}{|c|c|}
\hline ID & Description \\
\hline GO:0005524 & ATP binding \\
\hline GO:0003682 & chromatin binding \\
\hline GO:0008301 & DNA binding, bending \\
\hline
\end{tabular}

\begin{tabular}{|c|c|}
\hline \multicolumn{1}{|c|}{ ID } & Description \\
\hline GO:0090307 & Mitotic spindle assembly \\
\hline GO:0007080 & Mitotic metaphase plate congression \\
\hline GO:0031536 & Positive regulation of exit from mitosis \\
\hline GO:0000070 & Mitotic sister chromatid segregation \\
\hline GO:0031577 & Spindle checkpoint \\
\hline
\end{tabular}

C Cell components



GO:0005654

\begin{tabular}{|l|r|}
\hline \multicolumn{1}{|c|}{ ID } & Description \\
\hline GO:0005634 & nucleus \\
\hline GO:0005737 & cytoplasm \\
\hline GO:0005654 & nucleoplasm \\
\hline GO:0030496 & midbody \\
\hline GO:0016020 & membrane \\
\hline
\end{tabular}

D

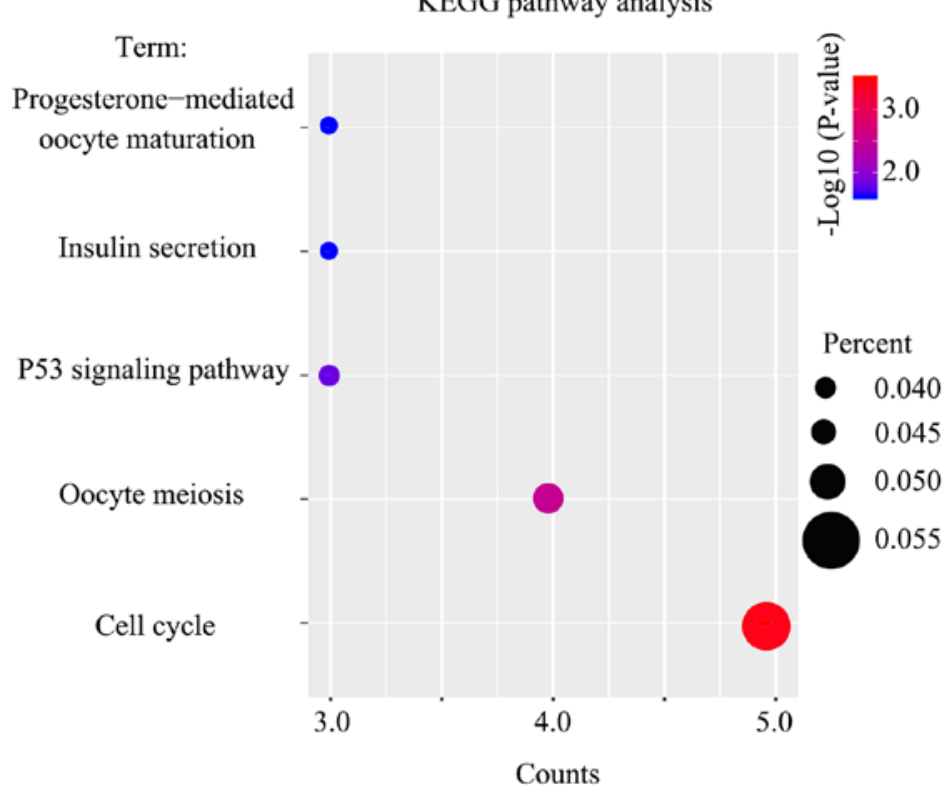

Figure 5. GO and KEGG analysis. (A-C) GO analysis for core genes. (D) KEGG analysis for core genes. GO, Gene Ontology; KEGG, Kyoto Encyclopedia of Genes and Genomes.

staining was performed in 23 intrahepatic cholangiocarcinoma tissues and corresponding adjacent tissues. The present study observed the expression of hub genes in intrahepatic cholangiocarcinoma tissues, adjacent normal liver tissues and intrahepatic bile duct tissues, and revealed that the expression of all hub genes was increased in most intrahepatic cholangiocarcinoma tissues compared with adjacent normal liver tissues and intrahepatic bile duct tissues (Table I; Fig. 8).

\section{Discussion}

Intrahepatic cholangiocarcinoma is the second most common primary liver malignant tumor globally, and cases of this disease are increasing annually. Unfortunately, the majority of patients are diagnosed at a nonsurgical stage and only $\sim 1$ in 5 cases are surgically resectable (15). However, a majority of patients are still at risk of tumor recurrence and drug resistance (16). Therefore, there is an urgent requirement to investigate the mechanisms of intrahepatic cholangiocarcinoma development, and the knowledge gained maybe useful in the development of novel clinical treatment strategies.

In the present study, a total of 1,643 real DEGs were identified and used to construct a WGCNA. Subsequent to analyzing the association between gene modules and clinical traits, brown and yellow modules were identified as key modules. A total of 63 core genes in the brown and yellow gene module were identified. The 63 core genes were highly enriched in mitotic spindle assembly (GO term), in ATP binding (GO term) and in the nucleus (GO term), and the pathways these genes were enriched in were the cell cycle pathway, oocyte meiosis 
A

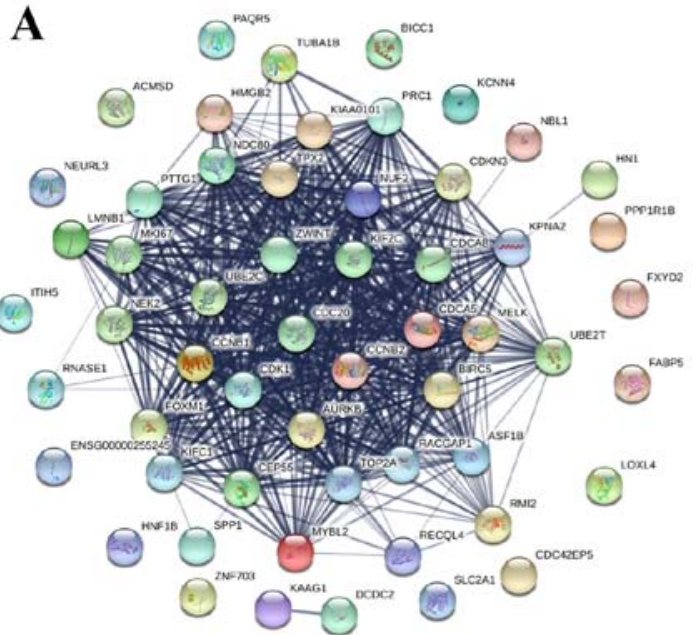

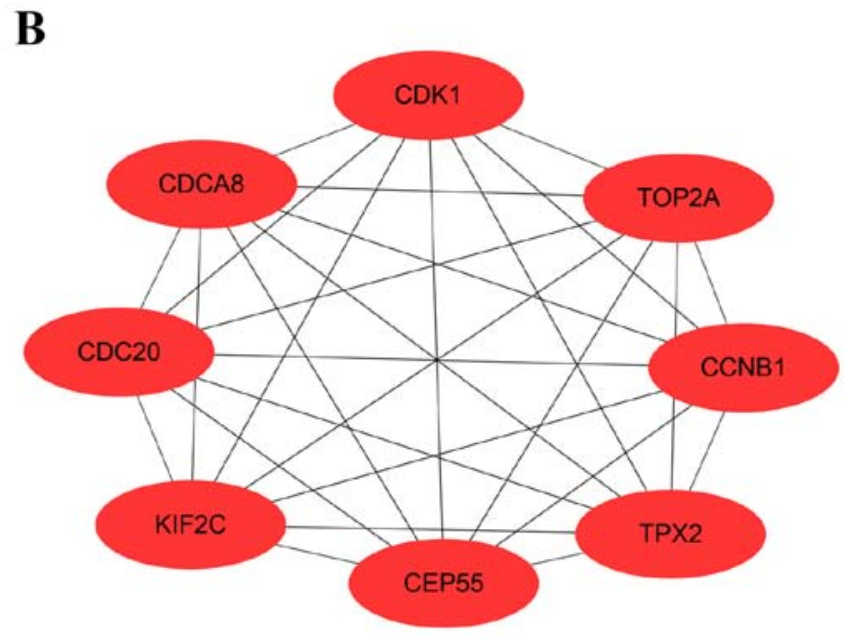

Figure 6. Construction of PPI and selection of hub genes. (A) Core genes were imported into STRING to construct a PPI network. Nodes indicate core genes, while edges indicate the correlation between core genes. (B) Cytoscape was used to analyze the PPI network, while hub genes were indicated by degree score and used to construct the PPI sub-network. PPI, protein-protein interactions; CCNB1, cyclin B1; CDC20, cell division cycle 20; CDCA8, cell division cycle associated 8; CDK1, cyclin dependent kinase 1; CEP55, centrosomal protein 55; KIF2C, kinesin family member 2C; TOP2A, DNA topoisomerase II $\alpha$; TPX2, TPX2 microtubule nucleation factor.
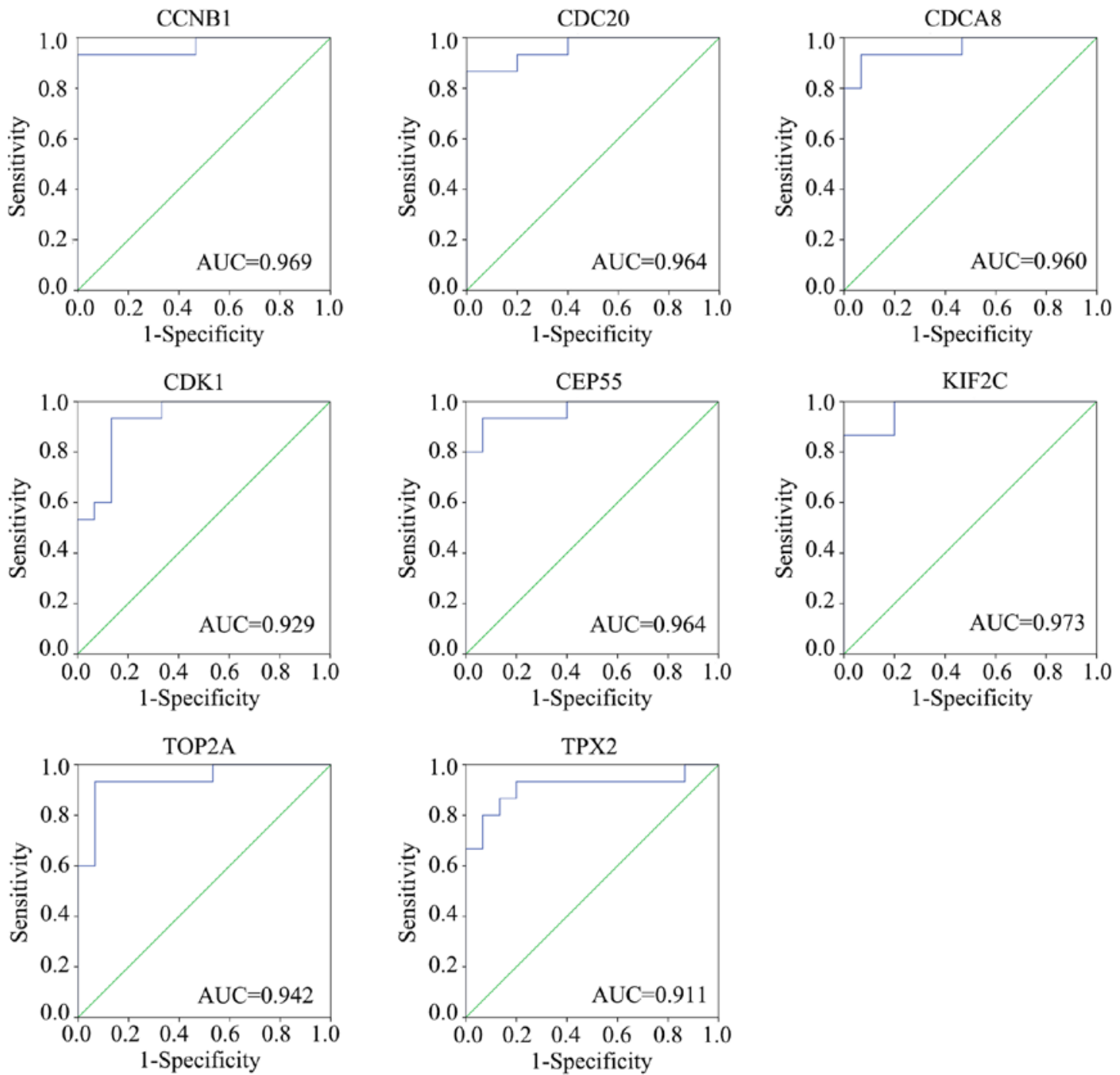

Figure 7. Verification of the diagnostic value of hub genes. Gene expression profile GSE119336 was obtained and receiver operating characteristic curve analysis was performed to identify the diagnostic value of hub genes CCNB1, CDC20, CDCA8, CDK1, CEP55, KIF2C, TOP2A and TPX2. CCNB1, cyclin B1; CDC20, cell division cycle 20; CDCA8, cell division cycle associated 8; CDK1, cyclin dependent kinase 1; CEP55, centrosomal protein 55; KIF2C, kinesin family member 2C; TOP2A, DNA topoisomerase II $\alpha$; TPX2, TPX2 microtubule nucleation factor. 
Table I. Analysis of the expression of targeted genes in intrahepatic cholangiocarcinoma tissues, adjacent liver tissues and intrahepatic bile duct tissues.

\begin{tabular}{|c|c|c|c|c|}
\hline Targets & Tissues & Low expression $(0-2)$ & Medium expression (3-4) & High expression (5-6) \\
\hline \multirow[t]{3}{*}{ CCNB1 } & Intrahepatic cholangiocarcinoma tissues & 2 & 5 & 16 \\
\hline & Adjacent liver tissues & 5 & 16 & 2 \\
\hline & Intrahepatic bile duct tissues & 20 & 2 & 1 \\
\hline \multirow[t]{3}{*}{ CDC20 } & Intrahepatic cholangiocarcinoma tissues & 1 & 4 & 18 \\
\hline & Adjacent liver tissues & 5 & 11 & 7 \\
\hline & Intrahepatic bile duct tissues & 17 & 4 & 2 \\
\hline \multirow[t]{3}{*}{ CDCA8 } & Intrahepatic cholangiocarcinoma tissues & 3 & 6 & 14 \\
\hline & Adjacent liver tissues & 9 & 11 & 3 \\
\hline & Intrahepatic bile duct tissues & 16 & 7 & 0 \\
\hline \multirow[t]{3}{*}{ CDK1 } & Intrahepatic cholangiocarcinoma tissues & 3 & 5 & 15 \\
\hline & Adjacent liver tissues & 4 & 16 & 3 \\
\hline & Intrahepatic bile duct tissues & 18 & 4 & 1 \\
\hline \multirow[t]{3}{*}{ CEP55 } & Intrahepatic cholangiocarcinoma tissues & 2 & 3 & 18 \\
\hline & Adjacent liver tissues & 7 & 15 & 1 \\
\hline & Intrahepatic bile duct tissues & 13 & 7 & 3 \\
\hline \multirow[t]{3}{*}{ KIF2C } & Intrahepatic cholangiocarcinoma tissues & 1 & 1 & 21 \\
\hline & Adjacent liver tissues & 8 & 12 & 3 \\
\hline & Intrahepatic bile duct tissues & 16 & 5 & 2 \\
\hline \multirow[t]{3}{*}{ TOP2A } & Intrahepatic cholangiocarcinoma tissues & 2 & 4 & 17 \\
\hline & Adjacent liver tissues & 6 & 13 & 4 \\
\hline & Intrahepatic bile duct tissues & 16 & 5 & 2 \\
\hline \multirow[t]{3}{*}{ TPX2 } & Intrahepatic cholangiocarcinoma tissues & 2 & 4 & 17 \\
\hline & Adjacent liver tissues & 3 & 16 & 4 \\
\hline & Intrahepatic bile duct tissues & 17 & 3 & 3 \\
\hline
\end{tabular}

CCNB1, cyclin B1; CDC20, cell division cycle 20; CDCA8, cell division cycle associated 8; CDK1, cyclin dependent kinase 1; CEP55, centrosomal protein 55 ; KIF2C, kinesin family member $2 \mathrm{C}$; TOP2A, DNA topoisomerase II $\alpha$; TPX2, TPX2 microtubule nucleation factor.

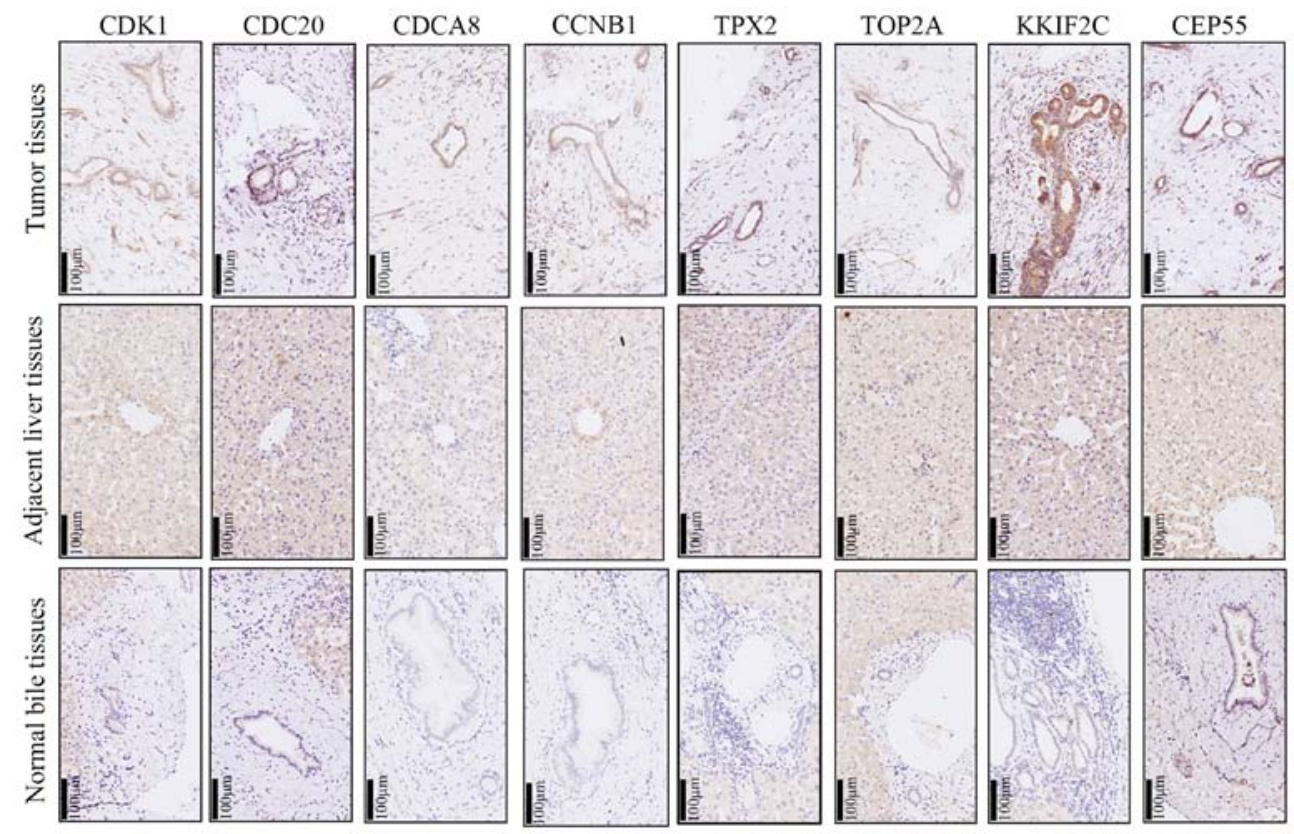

Figure 8. Representative figure of immumohistochemical staining for CCNB1, CDC20, CDCA8, CDK1, CEP55, KIF2C, TOP2A and TPX2 in intrahepatic cholangiocarcinoma tissues, adjacent liver tissues and intrahepatic bile duct tissues. CCNB1, cyclin B1; CDC20, cell division cycle 20; CDCA8, cell division cycle associated 8; CDK1, cyclin dependent kinase 1; CEP55, centrosomal protein 55; KIF2C, kinesin family member 2C; TOP2A, DNA topoisomerase II $\alpha$; TPX2, TPX2 microtubule nucleation factor. 
pathway, p53 signaling pathway, insulin secretion pathway and progesterone-mediated oocyte maturation pathway. Following the construction of the PPI network and the results of the ROC curve, a total of eight genes, including CCNB1, CDC20, CDCA8, CDK1, CEP55, KIF2C, TOP2A and TPX2 were identified as hub genes for intrahepatic cholangiocarcinoma. Furthermore, the protein expression levels of all these genes were increased in most intrahepatic cholangiocarcinoma tissues.

CCNB1 and CDK1 were indicated to be two genes that are able to directly regulate the cell cycle. CDK1 and CCNB1 proteins are located in the matrix of mitochondria. Here, they increase mitochondrial respiration, enhance oxygen consumption and promote ATP generation, which provides cells with enough bioenergy to complete the G2/M transition, and shortens the overall time to complete the cell cycle (17). Additionally, CDK1 and CCNB1 exhibit the potential to phosphorylate a number of pro-apoptotic and anti-apoptotic proteins, and are able to induce the inhibition of apoptosis in tumor cells (18). Furthermore, CDK1 and CCNB1 crosstalk with the $\mathrm{p} 53$ pathway, and activated CDK1 and CCNB1 are able to inhibit the effect of $\mathrm{p} 53$, and may result in cell proliferation and escape from apoptosis $(19,20)$. CDC20 is a downstream factor of the spindle assembly checkpoint and is a key co-factor of the anaphase-promoting complex or cyclosome E3 ubiquitin ligase (21). The potential of CDC20 as a therapeutic target for human cancer treatment has been reported in previous studies (22). Wu et al (23) investigated the effects of CDC20 overexpression in docetaxel- and castration-resistant prostate cancer cell lines, and demonstrated that CDC20 mediated resistance in a Bim-dependent manner. Paul et al (24) indicated that CDC20 regulated the proteasome-mediated degradation of the tumor suppressor BTG3 associated nuclear protein, and inhibited apoptosis in breast cancer cells. Furthermore, Zhang et al (25) demonstrated that the inhibition of CDC20 using curcumin is a useful method in the treatment of patients with pancreatic cancer. CDCA8 is a component of the chromosomal passenger complex 52, which serves a vital function in mitosis and cell division (26). Previous studies have demonstrated that high CDCA8 expression is positively associated with a poor prognosis in a variety of cancer types, including in cutaneous melanoma (27) and bladder cancer (28). In breast cancer, the expression of CDCA8 has been demonstrated to be associated with disease-free survival rate (29). Additionally, CDCA8 exhibits the potential to promote breast cancer cell proliferation following stimulation with estrogen (30). However, the exact function of CDCA8 in intrahepatic cholangiocarcinoma has, to the best of our knowledge, not yet been determined.

CEP55 is located on chromosome 10q23.33, which is associated with cytokinesis. A variety of studies have demonstrated that CEP55 serves a key function in numerous biological processes within cancer cells, including in proliferation, migration and differentiation (31). The knockdown of CEP55 expression has been indicated to inhibit the proliferation of breast cancer cells (32). In lung adenocarcinoma, the high expression of CEP55 is negatively associated with patient overall survival rate (33). KIF2C is the most important member of the motor proteins family and serves a crucial function in spindle assembly and ensuring proper chromosome segregation during cell mitosis (34). The effect of KIF2C on the progression of cancer has been previously reported, and $\mathrm{KIF} 2 \mathrm{C}$ is an identified biomarker for the prognosis of human glioma (35). KIF2C has also been demonstrated to be overexpressed in primary breast cancer tissues and cell lines, and this may be suppressed by the ectopic introduction of p53 $(36,37)$. TOP2A encodes for a DNA topoisomerase, which exhibits the capacity to regulate the topologic states of DNA during transcription (38). TOP2A is overexpressed in nasopharyngeal carcinoma and is associated with a poor outcome (39). Additionally, high TOP2A expression in breast cancer is able to predict poor prognosis (40). Furthermore, TOP2A has been indicated to be highly expressed in $33 \%$ cases of patients with intrahepatic cholangiocarcinoma (41). TPX2 serves a key function in spindle formation and microtubule nucleation (42). High TPX2 expression in hepatocellular carcinoma has been associated with a poor prognosis and has been indicated to contribute to cell proliferation and metastasis (43). The inhibition of TPX2 may activate the p53 pathway and promote apoptosis in breast cancer (44).

Through WGCNA, ROC analysis and immumohistochemical staining, the present study identified eight hub genes (CCNB1, CDC20, CDCA8, CDK1, CEP55, KIF2C, TOP2A and TPX2), which may contribute to the development of intrahepatic cholangiocarcinoma, and may also be novel biomarkers for the diagnosis of this disease. However, further research into the hub genes screened in the present study is urgently required to determine the underlying mechanisms associated with their function in intrahepatic cholangiocarcinoma.

\section{Acknowledgements}

Not applicable.

\section{Funding}

The present study was supported by the Program For Tackling Key Problems in Science and Technology (grant. no. 2007AA301B35-2), the Natural Science Foundation of Hubei Province of China (grant. no. 2010CDB06807) and the Important Project of Wuhan Administration of Science and Technology (grant. no. 2.008E+11).

\section{Availability of data and materials}

The datasets used and/or analyzed during the present study are available from the corresponding author on reasonable request.

\section{Authors' contributions}

$\mathrm{ZY}, \mathrm{ZZ}$ and DW were responsible for the data collection and analysis. ZC designed the study. SL and YS collected the clinical samples. ZY, ZZ, DW, SL and YS performed the experiments. $\mathrm{ZC}$ wrote the manuscript. All authors read and approved the final version of the article.

\section{Ethics approval and consent to participate}

All patients who had provided samples provided informed consent. The present study was approved by the Ethics 
Committee of the Renmin Hospital of Wuhan University (approval nr. WDRY2019-K075) and was performed in accordance with the Declaration of Helsinki.

\section{Patient consent for publication}

Not applicable.

\section{Competing interests}

The authors declare that they have no competing interests.

\section{References}

1. Chun YS and Javle M: Systemic and adjuvant therapies for intrahepatic cholangiocarcinoma. Cancer Control 24: $1073274817729241,2017$.

2. Nakano M, Ariizumi SI and Yamamoto M: Intrahepatic cholangiocarcinoma. Semin Diagn Pathol 34: 160-166, 2017.

3. Zhu Q, Sun Y, Zhou Q, He Q and Qian H: Identification of key genes and pathways by bioinformatics analysis with TCGA RNA sequencing data in hepatocellular carcinoma. Mol Clin Oncol 9: 597-606, 2018

4. Tang D, Zhao X, Zhang L, Wang Z and Wang C: Identification of hub genes to regulate breast cancer metastasis to brain by bioinformatics analyses. J Cell Biochem 120: 9522-9531, 2019.

5. Ma X, Tao R, Li L, Chen H, Liu Z, Bai J, Shuai X, Wu C and Tao K: Identification of a 5microRNA signature and hub miRNAmRNA interactions associated with pancreatic cancer. Oncol Rep 41: 292-300, 2019.

6. Xiao H, Xu D, Chen P, Zeng G, Wang X and Zhang X: Identification of five genes as a potential biomarker for predicting progress and prognosis in adrenocortical carcinoma. J Cancer 9 : 4484-4495, 2018.

7. Huang Y, Huang Y, Zhang L, Chang A, Zhao P, Chai X and Wang J: Identification of crucial genes and prediction of small molecules for multidrug resistance of Hodgkin's lymphomas. Cancer Biomark 23: 495-503, 2018.

8. Edgar R, Domrachev $M$ and Lash AE: Gene Expression Omnibus: NCBI gene expression and hybridization array data repository. Nucleic Acids Res 30: 207-210, 2002

9. Zhang K, Yu J, Yu X, Han Z, Cheng Z, Liu F and Liang P: Clinical and survival outcomes of percutaneous microwave ablation for intrahepatic cholangiocarcinoma. Int J Hyperthermia 34: 292-297, 2018.

10. Meng ZW, Pan W, Hong HJ, Chen JZ and Chen YL: Modified staging classification for intrahepatic cholangiocarcinoma based on the sixth and seventh editions of the AJCC/UICC TNM staging systems. Medicine (Baltimore) 96: e7891, 2017.

11. Huang da W, Sherman BT and Lempicki RA: Systematic and integrative analysis of large gene lists using DAVID bioinformatics resources. Nat Protoc 4: 44-57, 2009.

12. Huang da W, Sherman BT and Lempicki RA: Bioinformatics enrichment tools: Paths toward the comprehensive functional analysis of large gene lists. Nucleic Acids Res 37: 1-13, 2009.

13. Szklarczyk D, Gable AL, Lyon D, Junge A, Wyder S, Huerta-Cepas J, Simonovic M, Doncheva NT, Morris JH, Bork P, et al: STRING v11: Protein-protein association networks with increased coverage, supporting functional discovery in genome-wide experimental datasets. Nucleic acids Res 47: D607-D613, 2019.

14. Shannon P, Markiel A, Ozier O, Baliga NS, Wang JT, Ramage D, Amin N, Schwikowski B and Ideker T: Cytoscape: A software environment for integrated models of biomolecular interaction networks. Genome Res 13: 2498-2504, 2003.

15. Wu L, Tsilimigras DI, Farooq A, Hyer JM, Merath K, Paredes AZ, Mehta R, Sahara K, Shen F and Pawlik TM: Potential survival benefit of radiofrequency ablation for small solitary intrahepatic cholangiocarcinoma in nonsurgically managed patients: A population-based analysis. J Surg Oncol 120: 1358-1364, 2019.

16. Laurent S, Verhelst X, Geerts A, Geboes K, De Man M, Troisi R, Vanlander A, Rogiers X, Berrevoet F and Van Vlierberghe H: Update on liver transplantation for cholangiocarcinoma: A review of the recent literature. Acta Gastroenterol Belg 82: 417-420, 2019
17. Wang Z, Fan M, Candas D, Zhang TQ, Qin L, Eldridge A, Wachsmann-Hogiu S, Ahmed KM, Chromy BA, Nantajit D, et al: Cyclin B1/Cdk1 coordinates mitochondrial respiration for cell-cycle G2/M progression. Dev Cell 29: 217-232, 2014.

18. Xie B, Wang S, Jiang N and Li JJ: Cyclin B1/CDK1-regulated mitochondrial bioenergetics in cell cycle progression and tumor resistance. Cancer Lett 443: 56-66, 2019.

19. Huskey NE, Guo T, Evason KJ, Momcilovic O, Pardo D, Creasman KJ, Judson RL, Blelloch R, Oakes SA, Hebrok M and Goga A: CDK1 inhibition targets the p53-NOXA-MCL1 axis, selectively kills embryonic stem cells, and prevents teratoma formation. Stem Cell Rep 4: 374-389, 2015.

20. Lu M, Breyssens H, Salter V, Zhong S, Hu Y, Baer C, Ratnayaka I, Sullivan A, Brown NR, Endicott J, et al: Restoring p53 function in human melanoma cells by inhibiting MDM2 and Cyclin B1/CDK1-phosphorylated nuclear iASPP. Cancer Cell 30: 822-823, 2016.

21. Kapanidou M, Curtis NL and Bolanos-Garcia VM: Cdc20: At the crossroads between chromosome segregation and mitotic exit. Trends Biochem Sci 42: 193-205, 2017.

22. Wang L, Zhang J, Wan L, Zhou X, Wang Z and Wei W: Targeting Cdc20 as a novel cancer therapeutic strategy. Pharmacol Ther 151: 141-151, 2015.

23. Wu F, Lin Y, Cui P, Li H, Zhang L, Sun Z, Huang S, Li S, Huang S, Zhao Q and Liu Q: Cdc20/p55 mediates the resistance to docetaxel in castration-resistant prostate cancer in a Bim-dependent manner. Cancer Chemother Pharmacol 81: 999-1006, 2018.

24. Paul D, Ghorai S, Dinesh US, Shetty P, Chattopadhyay S and Santra MK: Cdc20 directs proteasome-mediated degradation of the tumor suppressor SMAR1 in higher grades of cancer through the anaphase promoting complex. Cell Death Dis 8: e2882, 2017.

25. Zhang Y, Xue YB, Li H, Qiu D, Wang ZW and Tan SS: Inhibition of cell survival by curcumin is associated with downregulation of cell division cycle $20(\mathrm{Cdc} 20)$ in pancreatic cancer cells. Nutrients 9: E109, 2017.

26. Hindriksen S, Meppelink A and Lens SM: Functionality of the chromosomal passenger complex in cancer. Biochem Soc Trans 43: 23-32, 2015

27. Ci C, Tang B, Lyu D, Liu W, Qiang D, Ji X, Qiu X, Chen L and Ding W: Overexpression of CDCA8 promotes the malignant progression of cutaneous melanoma and leads to poor prognosis. Int J Mol Med 43: 404-412, 2019.

28. Bi Y, Chen S, Jiang J, Yao J, Wang G, Zhou Q and Li S: CDCA8 expression and its clinical relevance in patients with bladder cancer. Medicine (Baltimore) 97: e11899, 2018.

29. Phan NN, Wang CY, Li KL, Chen CF, Chiao CC, Yu HG, Huang PL and Lin YC: Distinct expression of CDCA3, CDCA5, and CDCA8 leads to shorter relapse free survival in breast cancer patient. Oncotarget 9: 6977-6992, 2018.

30. Bu Y, Shi L, Yu D, Liang Z and Li W: CDCA8 is a key mediator of estrogen-stimulated cell proliferation in breast cancer cells. Gene 703: 1-6, 2019.

31. Jeffery J, Sinha D, Srihari S, Kalimutho M and Khanna KK: Beyond cytokinesis: The emerging roles of CEP55 in tumorigenesis. Oncogene 35: 683-690, 2016.

32. Wang Y, Jin T, Dai X and Xu J: Lentivirus-mediated knockdown of CEP55 suppresses cell proliferation of breast cancer cells. Biosci Trends 10: 67-73, 2016.

33. Wu S, Wu D, Pan Y, Liu H, Shao Z and Wang M: Correlation between EZH2 and CEP55 and lung adenocarcinoma prognosis. Pathol Res Pract 215: 292-301, 2019

34. Ritter A, Kreis NN, Louwen F, Wordeman L and Yuan J: Molecular insight into the regulation and function of MCAK. Crit Rev Biochem Mol Biol 51: 228-245, 2015.

35. Bie L, Zhao G, Wang YP and Zhang B: Kinesin family member $2 \mathrm{C}(\mathrm{KIF} 2 \mathrm{C} / \mathrm{MCAK})$ is a novel marker for prognosis in human gliomas. Clin Neurol Neurosurg 114: 356-360, 2012.

36. Dai $X$, Hua $T$ and Hong $T$ : Integrated diagnostic network construction reveals a 4-gene panel and 5 cancer hallmarks driving breast cancer heterogeneity. Sci Rep 7: 6827, 2017.

37. Shimo A, Tanikawa C, Nishidate T, Lin ML, Matsuda K, Park JH, Ueki T, Ohta T, Hirata K, Fukuda M, et al: Involvement of kinesin family member $2 \mathrm{C} /$ mitotic centromere-associated kinesin overexpression in mammary carcinogenesis. Cancer Sci 99: 62-70, 2008.

38. Nelson WG, Haffner MC and Yegnasubramanian S: The structure of the nucleus in normal and neoplastic prostate cells: Untangling the role of type 2 DNA topoisomerases. Am J Clin Exp Urol 6: 107-113, 2018. 
39. Lan J, Huang HY, Lee SW, Chen TJ, Tai HC, Hsu HP, Chang KY and $\mathrm{Li} \mathrm{CF}$ : TOP2A overexpression as a poor prognostic factor in patients with nasopharyngeal carcinoma. Tumour Biol 35: 179-187, 2014

40. An X, Xu F, Luo R, Zheng Q, Lu J, Yang Y, Qin T, Yuan Z, Shi Y, Jiang $\mathrm{W}$ and Wang $\mathrm{S}$ : The prognostic significance of topoisomerase II alpha protein in early stage luminal breast cancer. BMC Cancer 18: 331, 2018

41. Potkonjak M, Miura JT, Turaga KK, Johnston FM, Tsai S, Christians KK and Gamblin TC: Intrahepatic cholangiocarcinoma and gallbladder cancer: Distinguishing molecular profiles to guide potential therapy. HPB (Oxford) 17: 1119-1123, 2015.

42. Neumayer G, Belzil C, Gruss OJ and Nguyen MD: TPX2: Of spindle assembly, DNA damage response, and cancer. Cell Mol Life Sci 71: 3027-3047, 2014.
43. Hsu CW, Chen YC, Su HH, Huang GJ, Shu CW, Wu TT and Pan HW: Targeting TPX2 suppresses the tumorigenesis of hepatocellular carcinoma cells resulting in arrested mitotic phase progression and increased genomic instability. J Cancer 8: 1378-1394, 2017.

44. Chen M,Zhang H, Zhang G, Zhong A, Ma Q, Kai J, Tong Y, Xie S, Wang Y, Zheng H, et al: Targeting TPX2 suppresses proliferation and promotes apoptosis via repression of the PI3k/AKT/P21 signaling pathway and activation of $\mathrm{p} 53$ pathway in breast cancer. Biochem Biophys Res Commun 507: 74-82, 2018.

This work is licensed under a Creative Commons Attribution-NonCommercial-NoDerivatives 4.0 International (CC BY-NC-ND 4.0) License. 